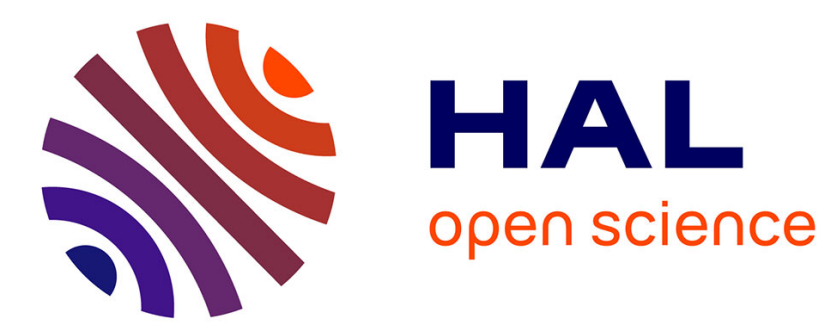

\title{
Optimal input design for a nonlinear dynamical uncertain aerospace system
}

Carine Jauberthie, Elodie Chanthery

\section{To cite this version:}

Carine Jauberthie, Elodie Chanthery. Optimal input design for a nonlinear dynamical uncertain aerospace system. IFAC Symposium on Nonlinear Control Systems, Sep 2013, Toulouse, France. hal-01966336

\section{HAL Id: hal-01966336 https://hal.laas.fr/hal-01966336}

Submitted on 28 Dec 2018

HAL is a multi-disciplinary open access archive for the deposit and dissemination of scientific research documents, whether they are published or not. The documents may come from teaching and research institutions in France or abroad, or from public or private research centers.
L'archive ouverte pluridisciplinaire HAL, est destinée au dépôt et à la diffusion de documents scientifiques de niveau recherche, publiés ou non, émanant des établissements d'enseignement et de recherche français ou étrangers, des laboratoires publics ou privés. 


\title{
Optimal input design for a nonlinear dynamical uncertain aerospace system
}

\author{
C. Jauberthie ${ }^{1,2}$ E. Chanthery ${ }^{1,3}$ \\ ${ }^{1}$ LAAS, CNRS, 7 avenue du Colonel Roche, F-31400 Toulouse, \\ France (cjaubert,echanthe@laas.fr) \\ ${ }^{2}$ Université de Toulouse, UPS, LAAS, F-31400 Toulouse, France \\ ${ }^{3}$ Université de Toulouse, INSA, LAAS, F-31400 Toulouse, France
}

\begin{abstract}
:
An optimal input design technique for aircraft uncertain parameter estimation is presented in this paper. The original idea is the combining of a dynamic programming method and interval analysis for the optimal input synthesis. This approach does not imply the estimation of a nominal value for parameter and allows to include realistic practical constraints on the input and output variables. The precise description of the approach is followed by an application in aerospace sciences.
\end{abstract}

\section{INTRODUCTION}

Experimental design is important for identifying mathematical models of modern aircraft dynamics from flight test data. The flight test input has a major impact on the quality of the data for modeling purposes. Good experimental design must account for practical constraints during the test. The overall goal is to design an experiment that produces data from which model parameters can be estimated accurately. Most importantly, in an estimation framework, the experimental conditions about noise and disturbances are usually properly modeled through appropriate assumptions about probability distributions (Mehra 1974], Walter et al. [1994], Kiefer [1974]). The conventional approach for the experimental design is based on stochastic models for uncertain parameters and measurement errors (see for example Roja et al. 2006]). However, other sources of uncertainty are not well-suited to the stochastic approach and are better modeled as bounded uncertainty. This is the case of parameter uncertainties that generally arise from design tolerances and from aging. In such cases, combining stochastic and bounded uncertainties may be an appropriate solution. Motivated by the above observations, we consider the optimal input design problem for nonlinear dynamical models with bounded uncertainties on parameters and measurement noise modeled by a gaussian distribution. Some works consider that the parameters belong to some prior domain, on which no probability function has to be defined (for example Pronzato et al. [1988], Belforte et al. [2004]). They aim at optimizing the worst possible performance of the experiment over the prior domain for the parameters (Pronzato et al. [1988]). In Pronzato et al. [1988], this maximin approach to synthesis the optimal input is described and the specific criterion are developped.

In this paper, it is supposed that the uncertainty on parameters can be modelled by bounded intervals and we use the concepts of interval analysis for the optimal input synthesis. The original approach of optimal input design for uncertain bounded parameter estimation described here is an extension of the works of E.A. Morelli (Morelli [1999]) using the dynamic programming. In the presented approach, the concepts of dynamical programming are combined with the maximin approach and with the tools of interval analysis.

The nonlinear controlled dynamic models considered in this paper can be written as:

$$
\left\{\begin{array}{l}
\dot{x}(t, p)=f(x(t, p), p)+u(t) g(x(t, p), p) \\
y(t, p)=h(x(t, p), p) \\
x(0)=x_{0} \in X_{0}, \quad p \in P \subset \mathcal{U}_{\mathcal{P}}
\end{array}\right.
$$

In these equations, the initial conditions $x_{0}$ are supposed to belong to a bounded set $X_{0}$. The input function $u$ is assumed to be piecewise continuous or differentiable. $x(t, p) \in \mathbb{R}^{n}$ and $y(t, p) \in \mathbb{R}^{m}$ denote respectively the state variables and the measured outputs. The vector of parameters $p$ belongs to a bounded connected set of parameters $P$ and $P$ is supposed to belong to $\mathcal{U}_{\mathcal{P}}$ where $\mathcal{U}_{\mathcal{P}}$ is an a priori known set of admissible parameters. $\mathcal{U}_{\mathcal{P}}$ is either included in $\mathbb{R}^{l}$ or equal to $\mathbb{R}^{l}$. The time interval is $\left[0, t_{\max }\right]$. The functions $f(x, p), g(x, p)$ and $h(x, p)$ are real and analytic on $M$ for every $p \in P$ ( $M$ is a connected open subset of $\mathbb{R}^{n}$ such that $x(t, p) \in M$ for every $p \in P$ and every $\left.t \in\left[0, t_{\max }\right]\right)$. The single-input case is considered for notational simplicity; however, all the results can be generalized.

The measurement data $z$ are assumed to be given by:

$$
z\left(t_{i}\right)=y\left(t_{i}, p\right)+\nu\left(t_{i}\right), \quad i=1, \ldots, N,
$$

where the measurement noise $\nu\left(t_{i}\right)$ is assumed white gaussian with zero mean and $E\left[\nu\left(t_{i}\right) \nu^{\top}\left(t_{j}\right)\right]=R \delta_{i j}$ where $R$ is the measurement noise covariance matrix and $i, j=$ $1, \ldots, N$. We suppose the matrix $R$ is known by physical experiments and sensor knowledge. The test duration $T$ is assumed to be fixed and such that $T \leq t_{\max }$.

In the case of aerospace models, constraints arising from practical flight test considerations were imposed on all input amplitudes and selected output amplitudes. Control surface amplitudes are limited by mechanical stops, flight 
control software limiters, or linear control effectiveness. Selected output amplitudes must be limited to avoid departure from the desired flight test condition and to ensure validity of the model. In addition, constraints may be required on aircraft attitude angles for flight test operational considerations, such as flight safety. In our case, the constraints are specified by:

$$
\begin{aligned}
\left|u(t)-u_{0}\right| & \leq \mu, \forall t \in\left[0, t_{\max }\right], \\
\left|y_{k}(t, p)-y_{k_{0}}\right| & \leq \eta_{k}, \forall t, k=1,2, \ldots, m,
\end{aligned}
$$

where $\mu$ and $\eta_{k}$ are positive constants, $u_{0}$ and $y_{k_{0}}$ are the trim values of $u$ and $y_{k}$.

In this paper, only the case of an aerospace model with a piecewise constant input is considered. Nevertheless the proposed approach can be easily applied to most of the dynamical models with constraints on inputs and eventually outputs.

This paper is organized as follows. In Section 2, the developped approach based on principles of dynamic programming and interval analysis is detailed. Optimal input functions are then computed. In Section 3, this approach is applied to the input design for the identification of an aircraft model. In this section, numerical results are proposed to compare the estimation results obtained with an optimal input and a classical admissible input. Some concluding remarks and future works are proposed in Section 4.

\section{EXPERIMENTAL DESIGN}

In order to obtain optimal input and consequently the most accurate estimates of model parameters, the information content in the system output response during the test must be maximized.

The information contained in the response is embodied in the Fisher information matrix. As the measurement noise $\nu\left(t_{i}\right)$ is assumed white gaussian with zero mean and known covariance matrix (2), the Fisher information matrix elements are combinations of partial derivatives of the system response variables with respect to the model parameters (Fourgeaud et al. [1967, Sorenson 1980]). The Fisher information matrix elements and sensitivities are obtained by solving the so-called sensitivity equations. Input designs for parameter estimation experiments are evaluated by examining a scalar function of the Fisher information matrix, denoted $F(p, \Xi)$ where $\Xi$ corresponds to the experimental conditions. In our case $\Xi$ represents parameters which characterize the input. $F(p, \Xi)$ is given by, $\forall p \in P$ :

$$
F(p, \Xi)=\sum_{i=1}^{N}\left(\frac{\partial y\left(t_{i}, p\right)}{\partial p}\right)^{\top} R^{-1}\left(\frac{\partial y\left(t_{i}, p\right)}{\partial p}\right)
$$

where the sensitivities $\frac{\partial y\left(t_{i}, p\right)}{\partial p}$ are solutions of:

$$
\begin{aligned}
\frac{d}{d t}\left(\frac{\partial x}{\partial p_{j}}\right)= & \sum_{k=1}^{n}\left[\frac{\partial f}{\partial x_{k}} \frac{\partial x_{k}}{\partial p_{j}}\right]+\frac{\partial f}{\partial p_{j}} \\
& +u(t)\left(\sum_{k=1}^{n}\left[\frac{\partial g}{\partial x_{k}} \frac{\partial x_{k}}{\partial p_{j}}\right]+\frac{\partial g}{\partial p_{j}}\right)
\end{aligned}
$$

$$
\frac{\partial y}{\partial p_{j}}=\sum_{k=1}^{n}\left[\frac{\partial h}{\partial x_{k}} \frac{\partial x_{k}}{\partial p_{j}}\right]+\frac{\partial h}{\partial p_{j}}, j=1, \ldots, l .
$$

In the problem of optimal input design for a nonlinear system, the optimal experiment as classically defined via the Fisher information matrix depends on the parameter estimate (Walter et al. 1994]) which is unknown before estimation. A classical approach consists in giving a nominal parameter value and finding the optimal experimental conditions for this value. Another approach takes into account the uncertainty on the nominal value (for more information, see Walter et al. [1994] or Jauberthie 2002] chapter four).

The optimal input design methodology that we have developed takes into account some bounded intervals for each parameter to be estimated. These intervals can model for example the uncertainty on the nominal value. Furthermore, the Fisher information matrix will be numerically computed for all $p$ in the bounded connected set $P$. In fact, by using the tools of interval analysis, the Fisher information matrix will be computed for the bounded connected set $P$. Thus, if we suppose that the bounded connected set $P$ is given by a cartesian product of intervals, the Fisher information matrix is an interval matrix and the obtained cost function to be optimised is given by an interval.

In this section, we first recall some classical concepts of interval analysis, mainly taken from Moore [1959] and Jaulin et al. 2001]. Then the adopted approach for the input design synthesis is presented. The third subsection gives our method for parameter estimation by using weighted least squares.

\subsection{Some concepts of interval analysis}

Interval analysis was initially developed to account for the quantification errors introduced by the rational representation of real numbers in computers and was extended to validated numerics (Moore [1959]).

\subsubsection{Basic definitions and notations}

Definition 2.1. (Interval, width, midpoint). A real interval $[u]=[\underline{u}, \bar{u}]$ is a closed and connected subset of $\mathbb{R}$ where $\underline{u}$ represents the lower bound of $[u]$ and $\bar{u}$ represents the upper bound. The width of an interval $[u]$ is defined by $w(u)=\bar{u}-\underline{u}$, and its midpoint by $m(u)=(\bar{u}+\underline{u}) / 2$.

The set of all real intervals of $\mathbb{R}$ is denoted IR.

Definition 2.2. (Interval equality). Two intervals $[u]$ and $[v]$ are equal if and only if $\underline{u}=\underline{v}$ and $\bar{u}=\bar{v}$.

Real arithmetic operations are extended to intervals (Moore [1966]).

Arithmetic operations on two intervals $[u]$ and $[v]$ can be defined by:

$$
\circ \in\{+,-, *, /\},[u] \circ[v]=\{x \circ y \mid x \in[u], y \in[v]\} .
$$

Definition 2.3. (Interval vector). An interval vector (or box) $[X]$ is a vector with interval components and may equivalently be seen as a cartesian product of scalar intervals:

$$
[X]=\left[x_{1}\right] \times\left[x_{2}\right] \times \ldots \times\left[x_{n}\right]
$$


with $\left[x_{i}\right]$ in IR, $i=1, \ldots, n$. The set of $n$-dimensional real interval vectors is denoted by $\mathrm{IR}^{n}$.

Definition 2.4. (Interval matrix). An interval matrix is a matrix with interval components.

The set of $n \times m$ real interval matrices is denoted by $\mathrm{IR}^{n \times m}$.

Definition 2.5. (width, midpoint). The width $w($.$) of an$ interval vector (or of an interval matrix) is the maximum of the widths of its interval components. The midpoint $m($.$) of an interval vector (resp. an interval matrix) is a$ vector (resp. a matrix) composed of the midpoint of its interval components.

Classical operations for interval vectors (resp. interval matrices) are direct extensions of the same operations for real vectors (resp. real matrices) (Moore [1966]).

Definition 2.6. (range). Let $f: \mathbb{R}^{n} \rightarrow \mathbb{R}^{m}$, the range of the function $f$ over an interval vector $[u]$ is given by:

$$
f([u])=\{f(x) \mid x \in[u]\} .
$$

The interval function $[f]$ from $\operatorname{IR}^{n}$ to $\operatorname{IR}^{m}$ is an inclusion function for $f$ if:

$$
\forall[u] \in \operatorname{IR}^{n}, \quad f([u]) \subseteq[f]([u]) .
$$

Property 2.1. An inclusion function of $f$ can be obtained by replacing each occurrence of a real variable by its corresponding interval and by replacing each standard function by its interval evaluation.

Such a function is called the natural inclusion function. In practice the inclusion function is not unique, it depends on the syntax of $f$.

The following subsection concerns the integration of ordinary differential equations with bounded uncertainties (like for example the first equation of (1) or (6)). Thus, the aim of this subsection is to estimate the sensitivities $\frac{\partial x}{\partial p}$ at the sampling times $\left\{t_{1}, t_{2}, \ldots, t_{N}\right\}$ corresponding to the measurement times of the outputs.

2.1.2 Integration of ordinary differential equations with bounded uncertainties When the differential equation is nonlinear like (1) or (6), the sets to be characterized may be nonconvex and may even consist of several disconnected components. The interval analysis consists in enclosing such sets in unions of nonoverlapping interval vectors and the usual drawback is to obtain wider and wider interval solution vectors. This is known as the wrapping effect. The wrapping effect leads to very pessimistic results.

The most effective methods to solve the state estimation for dynamical nonlinear systems are based on Taylor expansions (Berz et al. 1998, Moore 1959], Lohner [1987], Nedialkov et al. 2001b], Nedialkov et al. [2001a] or Rihm [1994], Raïssi et al. [2004]), Raïssi et al. [2006]. These methods consist in two parts. The first one verifies the existence and uniqueness of the solution by using the fixed point theorem and the Picard-Lindelof operator. An a priori box solution is computed. The second step consists in reducing the pessimism introduced in this a priori solution by using a high-order $k$ for the Taylor expansion and by using mean value forms (Neumaier 1990, Rihm [1994]) and matrices preconditioning.

\subsection{Optimal input design}

Our approach is based on dynamic programming principles. Dynamic programming allows practical constraints on the input and output variables to be included. Furthermore it is a very efficient method for performing a global exhaustive search.

The following cost function has been chosen:

$$
j(\Xi)=\operatorname{det}(F(p, \Xi)) \quad \forall p \in P .
$$

This criterion must be maximised (Walter et al. [1994]) by optimizing the input of the system.

Note that $F(p, \Xi)$ is positive definite thus all elements on the diagonal of $F(p, \Xi)$ are positive and the determinant of $F(p, \Xi)$ is positive. In the case of an interval matrix, this constraint must also hold. If interval calculus generates intervals containing non positive values, these are spurious and may be removed. Thus a constraint is introduced which respects the properties of $F(p, \Xi)$ : each component of the diagonal of $F(p, \Xi)$ is positive. More information about satisfaction constraints can be found in Chabert [09].

In the following, the set $\mathcal{E}$ corresponds to the admissible experimental conditions. Thus the set $\mathcal{E}$ gives an admissible input set.

By using the concepts of interval analysis, it is possible to compute $j(\Xi)$ in $P$. Consequently, $j(\Xi)$ becomes an interval. Our goal is thus to found the value of $\Xi \in \mathcal{E}$ maximizing the upper bound of the criterion $j(\Xi)$.

In order to apply our method, the admissible input has been limited to full amplitude square waves only. In fact, analytic works for similar problems demonstrate that inputs similar to square waves were superior to sinusoidal inputs for parameter estimation (Chen [1975]). The method of dynamic programming leads to test splitting into stages: time is divided into discrete steps called stages. At each stage, the associated criterion and outputs are computed (for more details see Drevfus [1965] or Morelli [1999]). This technique has the advantage to discard any input among square wave sequence whose output trajectory exceeds constraint limits.

For example, if we suppose three positions $(-a, 0, a), a$ being a positive constant for a single-input on each stage and two stages, the inputs tested by this procedure are given by:

$$
u(t)=\sum_{i=0}^{1}\left(a \varepsilon_{i}-a \varepsilon_{i-1}\right) H\left(t-\tau_{i}\right), \varepsilon_{-1}=0,
$$

where $H$ is the Heaviside function. The variables $\tau_{i}$ are the switching times with $\tau_{0}$ the initial test time and $\varepsilon_{i} \in\{-1,0,+1\}(i=0,1)$.

More generally, the inputs tested by our procedure are given by:

$$
u(t)=u_{0}+\sum_{i=0}^{r}\left(a_{i} \varepsilon_{i}-a_{i-1} \varepsilon_{i-1}\right) H\left(t-\tau_{i}\right), \varepsilon_{-1}=0
$$

where $u_{0}$ is an input trim value. Indeed, the variables $a_{i}$ are chosen to be equal to the square wave full positive 
amplitude (Morelli [1999]). The given variables $\tau_{i}$ satisfy $\tau_{0}<\tau_{1}<\ldots<\tau_{r}$.

This step gives the optimal number of square waves (with fixed time and fixed amplitude) to be realized. The corresponding signal is an optimal square wave input obtained in a single-pass solution:

$$
\hat{u}(t)=u_{0}+\sum_{i=0}^{r}\left(a \hat{\varepsilon}_{i}-a \hat{\varepsilon}_{i-1}\right) H\left(t-\tau_{i}\right), \hat{\varepsilon}_{-1}=0 .
$$

An example of (11) is given on Figure 1.

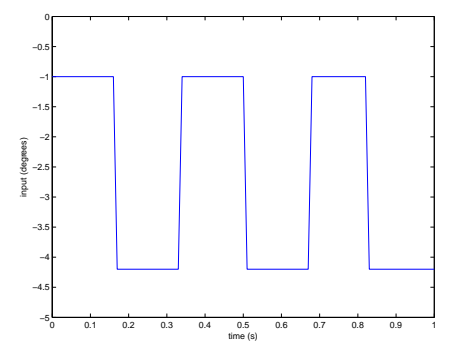

Fig. 1. Obtained input for six stages and $P_{5}$.

\subsection{Parameter estimation by weighted least squares}

The aim in this section is to propose a parameter estimation by using a classical least squares objective function based on the residuals at times $t_{i}$ and by exciting the system with the optimal input. This procedure uses the inverse of the measurement noise covariance matrix and gives an estimation of the parameter vector.

Let us consider a quadratic cost function $J(p)$ given by:

$$
J(p)=\sum_{i=1}^{N}\left(z\left(t_{i}\right)-y\left(t_{i}, p\right)\right)^{\top} R^{-1}\left(z\left(t_{i}\right)-y\left(t_{i}, p\right)\right) .
$$

The cost function is minimized with respect to the unknown parameter vector $p$ and leads to an estimated parameter vector denoted by $\hat{p}_{l s}$.

\section{EXAMPLE}

\subsection{The glider longitudinal motion}

The experimental design method is illustrated by an example concerning the glider longitudinal motion:

$$
\left\{\begin{aligned}
m \dot{V}= & -m g \sin (\theta-\alpha)-\frac{1}{2} \rho S V^{2}\left(C_{x}^{0}\right. \\
& \left.+C_{x \alpha}\left(\alpha-\alpha_{0}\right)+C_{x \delta_{m}}\left(\delta_{m}-\delta_{m_{0}}\right)\right) \\
m V(\dot{\alpha}-\dot{\theta})= & m g \cos (\theta-\alpha)-\frac{1}{2} \rho S V^{2}\left(C_{z}^{0}\right. \\
& +C_{z \alpha}\left(\alpha-\alpha_{0}\right)+C_{z q} \frac{q l}{V} \\
& \left.+C_{z \dot{\alpha}} \frac{\dot{\alpha} l}{V}+C_{z \delta_{m}}\left(\delta_{m}-\delta_{m_{0}}\right)\right) \\
B \dot{q}= & \frac{1}{2} \rho S l V^{2}\left(C_{m}^{0}+C_{m \alpha}\left(\alpha-\alpha_{0}\right)\right. \\
& +C_{m q} \frac{q l}{V}+C_{m \dot{\alpha}} \frac{\dot{\alpha} l}{V} \\
& \left.+C_{m \delta_{m}}\left(\delta_{m}-\delta_{m_{0}}\right)\right) \\
\dot{\theta}= & q .
\end{aligned}\right.
$$

In these equations, the state variables are given by $(V, \alpha, q, \theta)^{\top}$, the observations are $(V, \alpha, q, \theta)^{\top}$, the input $u$ to be designed is $\delta_{m}\left(u_{0}=\delta_{m_{0}}\right)$ and $\left(C_{z \dot{\alpha}}, C_{z q}, C_{m \dot{\alpha}}, C_{m q}\right)$ are the parameters to be identified.

The approach has been implemented in Matlab, using the toolbox Intlab (Rump 1999]). The initial conditions $x_{0}$ are supposed given by $(28.5,6.5,0,2.43)^{T}$.

The laboratory geometry and aircraft scale model lead to constraints on inputs and outputs:

$$
\begin{aligned}
& |u(t)+2.6| \leq 1.6 \mathrm{deg}, \quad\left(\delta_{m_{0}}=-2.6 \text { degrees }\right), \\
& 2 \mathrm{~m} \leq z\left(t_{f}\right) \leq 3 \mathrm{~m},
\end{aligned}
$$

where $z\left(t_{f}\right)$ represents the model altitude in the last seconds of flight (in meters). The flight test duration is fixed at one second (approximatively five seconds at full scale). Experimental conditions are given by $\Xi_{1}=$ $\left(\varepsilon_{0}, \ldots, \varepsilon_{r}\right)$ and

$$
\mathcal{E}=\left\{\Xi_{1} \in \mathbb{R}^{r+1} \mid \varepsilon_{i} \in\{-1,0,+1\}, i=0, \ldots, r\right\} .
$$

\subsection{Optimal input design}

To make dynamic programming applicable, the flight test is split into stages. In order to avoid a long computational time, the flight test is split into respectively two, three, four, five and six stages. Consequently the input 3211 (Mulder et al. [1994]) cannot be obtained by the developped software.

For each stage, the Fisher information matrix (5) is computed with $R$ given by a diagonal matrix:

$$
R=\left[\begin{array}{cccc}
25.10^{-4} & 0 & 0 & 0 \\
0 & 4.10^{-2} & 0 & 0 \\
0 & 0 & 4.10^{-2} & 0 \\
0 & 0 & 0 & 4.10^{-2}
\end{array}\right]
$$

The initial parameter box contains a nominal value computed by wind tunnel experiments: $p_{0}=(1.8,5,-5,-22)$ (Jauberthie [2002]).

In this work, we introduce an uncertainty around this nominal value from $1 \%, 5 \%$ or $10 \%$ thus the boxes of parameter will be:

$$
\begin{gathered}
P_{1}=\left[\begin{array}{cc}
1.782 & 1.818 \\
4.950 & 5.050 \\
-5.050 & -4.950 \\
-22.220 & -21.780
\end{array}\right], P_{5}=\left[\begin{array}{cc}
1.710 & 1.890 \\
4.750 & 5.250 \\
-5.250 & -4.750 \\
-23.100 & -20.900
\end{array}\right], \\
\text { and } \mathrm{P}_{10}=\left[\begin{array}{lr}
1.620 & 1.980 \\
4.500 & 5.500 \\
-5.500 & -4.500 \\
-24.200 & -19.800
\end{array}\right] .
\end{gathered}
$$

The obtained results will be compared with those obtained without uncertainty around $p_{0}$.

To compute the solutions of (13), we use the method presented in subsection 2.1.2.

The obtained input (for six stages) and $P_{5}$ is the following:

$$
\begin{aligned}
\hat{u}_{6}(t)= & \delta_{m 0}+a_{6_{0}} H\left(t-\tau_{0_{6}}\right)-2 a_{6_{1}} H\left(t-\tau_{1_{6}}\right) \\
& +2 a_{6_{2}} H\left(t-\tau_{2_{6}}\right)-2 a_{6_{3}} H\left(t-\tau_{3_{6}}\right) \\
& +2 a_{6_{4}} H\left(t-\tau_{4_{6}}\right)-2 a_{65} H\left(t-\tau_{5_{6}}\right),
\end{aligned}
$$

with: $a_{6_{i}}=1.6(i=1, \ldots, 5)$ degrees, $\tau_{0_{6}}=0 \mathrm{~s}, \tau_{1_{6}}=$ $0.1667 \mathrm{~s}, \tau_{2_{6}}=0.3334 \mathrm{~s}, \tau_{3_{6}}=0.5001 \mathrm{~s}, \tau_{4_{6}}=0.6668 \mathrm{~s}$, 
$\tau_{5_{6}}=0.8335 \mathrm{~s}$. The input trajectory corresponding to (16) is given on the Figure 1.

The obtained input for six stages and $P_{10}$ is the same as previous one.

Tables 1 and 2 show the criterion values obtained after running the software for two, three, four, five and six stages.

\begin{tabular}{ccc}
\hline Number of stages & $p_{0}$ & $P_{1}$ \\
\hline two & $3.3792 \times 10^{-8}$ & 0.2302 \\
three & $4.5902 \times 10^{-7}$ & $8.3146 \times 10^{3}$ \\
four & $6.2648 \times 10^{-6}$ & $1.8851 \times 10^{3}$ \\
five & $1.5011 \times 10^{-4}$ & $3.8774 \times 10^{6}$ \\
six & $7.1596 \times 10^{-2}$ & $1.7746 \times 10^{10}$ \\
\hline
\end{tabular}

Table 1. Values of the cost function $\operatorname{det}(F(P, \Xi))$ for optimal experiment design.

\begin{tabular}{ccc}
\hline Number of stages & $P_{5}$ & $P_{10}$ \\
\hline two & 2.7052 & 15.0854 \\
three & $3.2382 \times 10^{5}$ & $7.6249 \times 10^{6}$ \\
four & $2.2809 \times 10^{8}$ & $1.0890 \times 10^{11}$ \\
five & $3.8515 \times 10^{12}$ & $3.4819 \times 10^{15}$ \\
six & $2.4661 \times 10^{16}$ & $3.0662 \times 10^{19}$ \\
\hline
\end{tabular}

Table 2. Values of the cost function $\operatorname{det}(F(P, \Xi))$ for optimal experiment design.

It is shown that the width of the obtained intervals increases with the parameter uncertainty and the obtained results given with $P_{10}$ are high. The width of intervals increases also with the number of stages which is inherent to the interval calculation (Jaulin et al. [2001]).

To clearly present the benefits of this approach for parameter estimation, we use the method descibed in section 2.2 with two admissible inputs: a non-optimized input and an optimal input.

\subsection{Parameter estimation}

The following study was conducted as a simulation with the input $u=\hat{u}_{6}$. The simulated and measured output vector is given on Figures 2 and 3 .

Columns in Table 3 successively give the true parameter vector $\bar{p}$ which is $p_{0}$ and the estimated parameter vector $\hat{p}_{l s}$ as proposed in Section 2.3. The last column contains the relative errors as indicated.

\begin{tabular}{cccc}
\hline Parameter & $\bar{p}$ & $\hat{p}_{l s}$ & $\frac{\left|\hat{p}_{l s}-\bar{p}\right|}{|\bar{p}|}$ \\
\hline$C_{z \dot{\alpha}}$ & 1.8 & 1.9435 & 0.0797 \\
$C_{z q}$ & 5 & 5.3157 & 0.0631 \\
$C_{m \dot{\alpha}}$ & -5 & -5.3961 & 0.0792 \\
$C_{m q}$ & -22 & -24.0142 & 0.0915 \\
\hline
\end{tabular}

Table 3. Estimates obtained with an optimized input.

Now, it is interesting to compare these results with those obtained from the following classical non-optimal input:

$$
u(t)=\left\{\begin{array}{l}
-2.6 \text { degrees } 0 \mathrm{~s} \leq t \leq 0.25 \mathrm{~s} \\
-1 \text { degrees } 0.25 \mathrm{~s} \leq t \leq 0.5 \mathrm{~s} \\
-2.6 \text { degrees } 0.5 \mathrm{~s} \leq t \leq 1 \mathrm{~s}
\end{array}\right.
$$

In Table $4, \hat{p}$ represents the estimated parameter vector obtained with the estimation procedure given in Section 2.3 , by exciting the system with the previous input. The last column gives the relative errors as indicated.

\begin{tabular}{ccc}
\hline Parameter & $\hat{p}$ & $\frac{|\hat{p}-\bar{p}|}{\bar{p}}$ \\
\hline$C_{z \dot{\alpha}}$ & 0.4178 & 0.7678 \\
$C_{z q}$ & 8.1201 & 0.6240 \\
$C_{m \dot{\alpha}}$ & -6.2918 & 0.2584 \\
$C_{m q}$ & -24.8112 & 0.1278 \\
\hline
\end{tabular}

Table 4. Estimates obtained with a nonoptimized input.

The optimal input improves the parameter estimation significantly. Clearly, the fourfth column of Table 3 and third column of Table 4 show an improvement in estimation results.

The trajectories presented in Figure 4 are obtained by solving (13) with $u=\hat{u}_{6}, p=\bar{p}$ (full line) and $p=$ $\hat{p}_{l s}$ (dotted line). We can compare the reconstructions of the angle of attack and pitch rate. These reconstructions point out the efficiency of the proposed method.
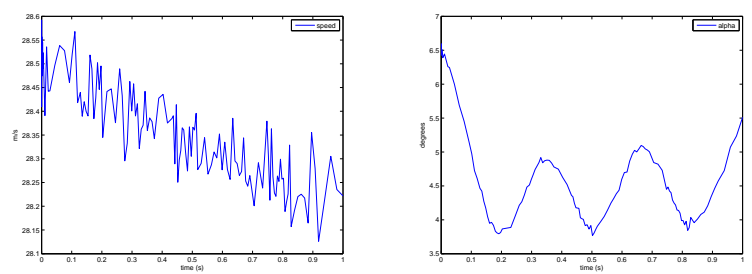

Fig. 2. Speed (left) / Angle of attack (right).
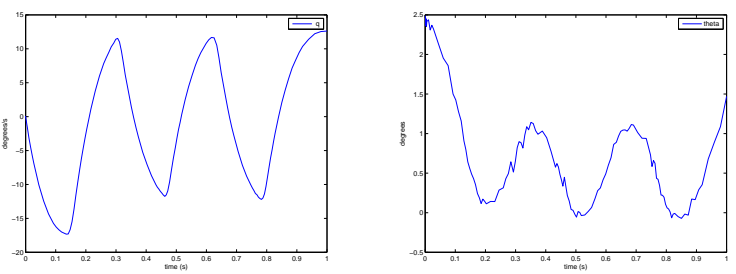

Fig. 3. Pitch rate (left) / Pitch angle (right).
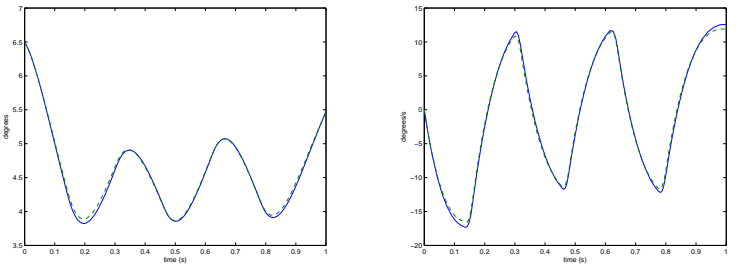

Fig. 4. Reconstruction of the angle of attack (in degree) (left) / Reconstruction of the pitch rate (in degree per second) (right). 


\section{CONCLUSION}

In this contribution, an efficient methodology for designing input for parameter estimation in aircraft systems is given. The developped method has potential for producing practical, suitable solutions for input design problems of current interest in aircraft parameter estimation flight experiments. It is suitable for multiple input design problems with output amplitude constraints, input design for control augmented aircraft for example. A comparison of the results between optimized tests and conventional tests highlights the advantages of the method proposed. The optimized test leads to better accuracy in estimation of coefficients. The proposed approach can be easily applied to most of the dynamical models with constraints on inputs and outputs.

Our future works concern an improvement in the estimation parameter problem for these models and the potential application of this method to the active diagnosis. In fact, this last objective will be to use these tools to achieve an active diagnostic methodology that is to find a sequence of actions to refine the diagnosis. Additional criteria based on the Fisher information matrix could be interesting (more information could be found in Kiefer [1974] or Jauberthie 2002] chapter four).

\section{REFERENCES}

G. Belforte and P. Gay. Optimal worst case estimation for LPV-FIR models with bounded erros, Systems and Control Letters (53)259-268, 2004.

M. Berz and K. Makino. Verified integration of ODEs and flows using differential algebraic methods on high-order Taylor models, Reliable Computing, vol. 4(4), pp 361369, 1998.

G. Chabert and L. Jaulin. Contractor programming, Artifical Intelligence, vol. 173, pp 1079-1100, 2009.

R.T.N. Chen. Input design for aircraft parameter identification: using time optimal control formulation, AGARD-CP-172 paper 13, 1975.

G. Corliss. Guaranteed error bounds for ordinary differential equations, Lectures notes presented at the VI-th SERC Numerical Analysis Summer School, 1994.

S.E. Dreyfus. Dynamic programming and the Calculus of Variations. New York Academic Press, 1965.

C. Fourgeaud, A. Fuchs Statistique, Dunod, Paris, 1967.

C. Jauberthie. Planification d'expériences pour systèmes dynamiques, Thèse, Université de Technologie de Compiègne, 2002.

L. Jaulin, M. Kieffer, O. Didrit, E. Walter. Applied interval analysis, with examples in parameter and state estimation, Robust control and robotics, Springer, Londres, 2001.

J. Kiefer. General equivalence theory for optimum designs (approximate theory), Annals of stat. (2)5 pp 849-879, 1974.

R. Lohner. Enclosing the solution of ordinary initial and boundary value problems, in E. Kaucher, U. Kulisch, and C. Ullrich (eds) Computer arithmetic: Scientific Computation and Programming Languages, BG Teubner, Stuttgart, Germany, pp 255-286, 1987.

R.K. Mehra, Optimal input signals for parameter estimation in dynamic systems - Survey and new results, IEEE Vol.AC-19, No. 6, 1974.
M. Milanese, J.P. Norton, H. Piet-Lahanier and E. Walter, Bounding approaches to system identification, Plenum, New York; 1996.

M. Milanese and A, Vivino. Estimation theory for nonlinear models and set membership uncertainty, Automatica, vol. 27, no. 2, pp 403-408, 1991.

R.E. Moore. Automatic error analysis in digital computation, Technical report LMSD-48421, Lockheed Missiles and Space Co, Palo Alto, CA; 1959.

R.E. Moore. Interval Analysis, Prentice Hall, New Jersey; 1966.

E.A. Morelli, Flight test of optimal inputs and comparison with conventional inputs, Journal of Aircraft (36)2 (1999).

J.A. Mulder, J.K. Sridhar, J.H. Breeman. Identification of dynamic system-application to aircraft nonlinear analysis and manoeuvre design. Technical Report AG 300, AGARD, Vol. 9, Part 2, 1994.

N.S. Nedialkov and K.R. Jackson Methods for initial value problems for ordinary differential equations, In $U$. Kulisch, R. Lohner and A. Facius, editors, Perspectives on Enclosure Methods, Springer Verlag, Vienna, pp 219$264,2001$.

N.S. Nedialkov, K.R. Jackson, J.D. Pryce. An effective high-order interval method for validating existence and uniqueness of the solution of an IVP for an ODE, Reliable Computing, vol 7(6), pp 449-465, 2001.

A. Neumaier. Interval Methods for Systems of Equations, Cambridge University Press, Cambridge, UK; 1990.

L. Pronzato, E. Walter. Robust experiment design via maximin optimization, Mathematical Biosciences, (89)161-176, 1988.

T. Raïssi, N. Ramdani, Y. Candau. Robust nonlinear continuous-time state estimation using interval taylor models. Proceedings of the 6th IFAC Symposium on Robust Control, Toulouse, France, pp 691-696, 2006.

T. Raïssi, N. Ramdani, Y. Candau. Set membership state and parameter estimation for systems described by nonlinear differential equations, Automatica, vol 40, pp 1771-1777, 2004

R. Rihm. Interval methods for initial value problems in ODEs, In J. Herzberger (Ed.), Topics in validated computations: Proceedings of the IMACS-GAMM international workshop on validated computations, University of Oldenburg, Elsevier Studies in Computational Mathematics, Amsterdam, New York: Elsevier, 1994.

C.R. Rojas, J.S. Welsh, G.C. Goodwin, A. Feuer.Robust optimal experiment design for system identification, Automatica (43)993-1008, 2006.

S.M. Rump. INTLAB - INTerval LABoratory. In Tibor Csendes, editor, Developments in Reliable Computing, pages 77-104. Kluwer Academic Publishers, Dordrecht, 1999. http://www.ti3.tuhh.de/rump/.

H.W. Sorenson, Parameter estimation, Principles and Problems, Marcel Dekker, New York, 1980.

E. Walter, L. Pronzato, Identification de modèles paramétriques à partir de données expérimentales, MASSON, 1994. 